\title{
Is WR 103 (WC9) as violent as the WN8 stars?
}

Pieter M. Veen ${ }^{1}$, Arnout M. van Genderen ${ }^{1}$, Karel A. van der Hucht ${ }^{2}$, and Chris Sterken ${ }^{3}$

\author{
${ }^{1}$ Leiden Observatory, PO Box 9513, NL-2300 RA Leiden, \\ the Netherlands \\ ${ }^{2}$ SRON, Sorbonnelaan 2, NL-3584 CA Utrecht, the Netherlands \\ ${ }^{3}$ University of Brussels (VUB), Pleinlaan 2, B-1050 Brussels, Belgium
}

\section{Photometry}

We collected photometric data of WR 103 (HD 164270, WC9), both in the Walraven system and in the Strömgren system. Figure 1 presents one intense monitoring run, performed in 1990 by $R$. van Ojik. We suggest to describe the light-curve as a saw-tooth with a time-scale of roughly 0.5 day. Alhough we confirm a cyclic light-variation of the object with an amplitude of $\sim 0.05$ mag, our data do not support the period of $P=0.88 \mathrm{~d}$ found by Isserstedt \& Moffat (1981) (see also Moffat et al. 1986; Balona et al. 1989).

The colour changes as displayed in Fig. 1 indicate that the star is blue when it is bright. The amplitude of the variation increases towards the violet. This suggests that we are dealing with continuum-light variations. Also the amplitude in the Strömgren b-band (not shown), suppressed by line-emission, supports this view. This behaviour is indicative of stellar pulsation, but the extrema are sharply peaked and do not seem to reproduce from night to night. Thus, at least it seems that pulsation cannot be the only explanation. Variable extinction, as observed during so called 'eclipses' caused by condensing dust clouds (Veen et al. 1998), cannot explain the $b$-band behaviour.

The continuum variation may be related to the so-called 'blobs' that are observed as moving spectral features on emission lines (Lépine et al. in these Proceedings). During two epochs we have obtained spectra simultaneously, that are not suitable to track the blobs. However, we do observe $E W$ variations of $5 \%$ and, notably, large variations of the P-Cygni absorption-throughs of, e.g., O III $\lambda 5592$.

\section{How does this relate to WN8 stars?}

Marchenko et al. (1998 and references therein) discuss intense monitoring of the all-variable 'violent' WN8 stars. They conclude that the following statistical trend exists: the larger the continuum variation (expressed as standard deviation), the more the P-Cygni absorption edge varies. However, a direct relationship has not been found and may vary from star to star. Qualitatively, WR 103 seems to behave similarly. In addition, Robert (1994) demonstrated that both WR 40 (WN8) and WR 103 showed the largest variability of C IV $\lambda 5807$ in a sample of nine stars. Furthermore, both objects also showed the largest polarimetric variability and the lowest mean number of inhomogeneities in the 


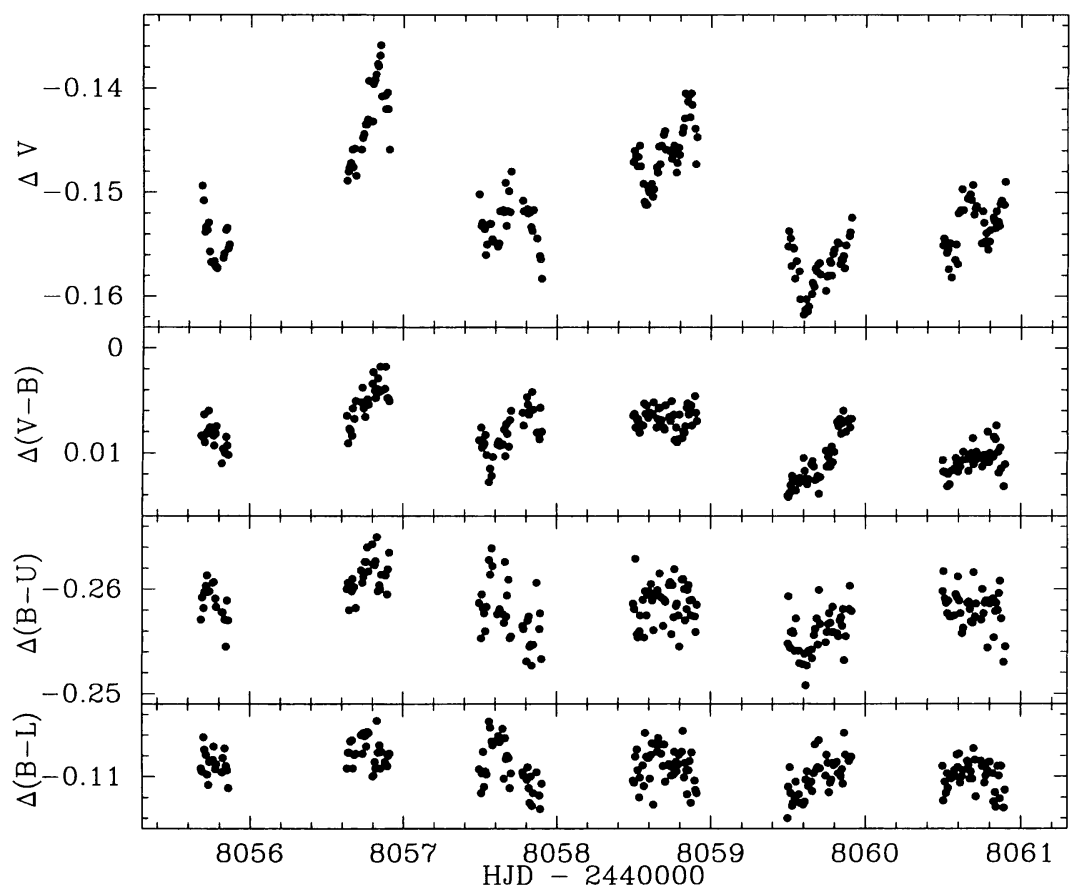

Figure 1. Multi-colour photometry in the Walraven system over six consecutive nights (log intensity scale).

wind. Another hint that WR 103 is comparable to WN8 stars, comes from spectropolarimetric data (Schulte-Ladbeck 1994). Again, both WR 40 and WR 103 are classified as locally inhomogeneous, but, if averaged over time, spherically symmetric.

We plan to investigate a possible correlation between our photometry and simultaneous spectroscopy, to compare the colour behaviour relative to the WN8 stars in our sample, and to examine whether 'blobs' can explain the continuum variations.

\section{References}

Balona, L.A., Egan, J., Marang, F. 1989, MNRAS 240, 103

Isserstedt, J., Moffat, A.F.J. 1981, A\&A 96, 133

Marchenko, S.V., Moffat, A.F.J., Eversberg, T. 1998, MNRAS 294, 642

Moffat, A.F.J., Lamontagne, R., Cerruti, M.A. 1986, PASP 98, 1170

Robert, C. 1994, in: A.F.J. Moffat, S.P. Owocki, A.W. Fullerton, N. St-Louis (eds.), Instability and Variability of Hot-Star Winds, ApSS 221, 137

Schulte-Ladbeck, R.E. 1994, ApSS 221, 347

Veen, P.M., van Genderen, A.M., van der Hucht, K.A., Li, A., Sterken, C., Dominik, C. 1998, A\&A 329,199 